\title{
FUTURE OBSERVATION OF THE F-CORONA WITH THE LASCO CORONOGRAPH SPACE EXPERIMENT
}

\author{
P.L. LAMY[1], A. LLEBARIA[1], A. MAUCHERAT[1] \\ S. KOUTCHMY[2], F. GIOVANE[3] \\ [1] Laboratoire d'Astronomie Spatiale \\ Les Trois Lucs, 13012 Marseille, France \\ [2] Institut d'Astrophysique de Paris \\ [3] University of Florida
}

\begin{abstract}
The Wide-field White Light and Spectrometric Coronograph (LASCO) to be flown on SOHO in 1995 will observe the corona from just above the limb at 1.1 out to 30 solar radii $\left(R_{0}\right)$. In addition to the fundamental problems of coronal physics (heating of the corona, acceleration of the solar wind, coronal transients), the scientific objectives incorporate the distribution and properties of dust particles including those released from sun-grazing comets, and interactions of coronal plasma with the dust.
\end{abstract}

\section{Introduction}

Most if not all of our knowledge of the F-corona come from ground-based, balloons and aircraft observations during total solar eclipses. The limitations of these observations are quite obvious, the available time is very short and the sky background limits the useful field-ofview to a few solar radii. Going to space has allowed to overcome the limitation imposed by the Earth's atmosphere: externally occulted coronographs have been implemented to reduce the now dominating instrumental scattered light. Surprisingly, space-born coronograph has produced no real advance in the field of interplanetary dust with the exception of Sun-grazing comets. To a large extent, the problem has to do with the poor photometric performances of the detectors used up to now (vidicons in particular). Even the elaborated Solar Maximum Mission coronograph with polarimetric capabilities suffered from this problem. As a consequence, quantitative data have seldom been obtained. The most valuable results were derived by image subtraction revealing the transitory plasma events in the corona.

The LASCO space coronograph will hopefully remedy to these shortcomings in particular thanks to its CCD cameras whose superior photometric performances are well established. It further has the capabilities of a large field-of-view $\left(30 R_{o}\right)$, of performing colorimetric, polarimetric measurements as well as high resolution spectrometric profiles. All these performances should enable LASCO to make a valuable investigation of the F-corona.

LASCO is a joint project of the Naval Research Laboratory which has the overall responsability of the instrument (P.I. G. Bruckner), the Max-Planck Institute für Aeronomie,

191

A.C. Levasseur-Regourd and H. Hasegawa (eds.), Origin and Evolution of Interplanetary Dust, 191-194.

(1) 1991 Kluwer Academic Publishers, Printed in Japan. 
Laboratoire d'Astronomie Spatiale and the University of Birmingham. It is part of the scientific payload of the SOHO spacecraft which is scheduled to be launched in July 1995 and to have an operational lifetime of at least two years. The late R. Giese participated as a coinvestigator in the definition of the scientific objectives of the instrument.

\section{Scientific objectives}

In addition to the fundamental problems of coronal physics (heating of the corona, acceleration of the solar wind, coronal transients), LASCO offers a unique opportunity to study the photopolarimetric/colorimetric properties of the F-corona (further required for the proper separation of the K-corona) and their connection to those of the Zodiacal Light, to study the dynamics of the circum-solar grains by Doppler shift and line profile measurements of a Fraunhofer line, to look for variations associated with solar coronal activity (CME's, solar flare associated shock waves, varying magnetic fields), to observe Sun-grazing comets or comets colliding with the Sun (adding a wealth of information on the dynamics of newly released dust).

The separation of the $\mathrm{K}$ and $\mathrm{F}$ components must be accurately accomplished to achieve the scientific goals. This is particularly accute in the outer corona where the $\mathrm{K}$ component represents only a few percent of the total brightness but is of fundamental interest for understanding the expansion of the solar wind.

Two different methods are used to achieve this separation: the spectroscopic method which works well up to $3 \mathrm{R}_{0}$ and the polarization method at larger distances. However, the classical approximation that the polarization of the F-corona is negligibly small no longer holds beyond about $5 \mathrm{R}_{\mathrm{o}}$. Clearly, the problem must be approached from the two fronts, namely by modeling the polarization of both the $\mathrm{K}$ and $\mathrm{F}$-corona, recalculating the polarized intensities and iterating until a satisfactory agreement is reached. A criterion of importance for the modeling of the F-corona is the continuity with the photopolarimetric properties of the Zodiacal Light.

\section{Description of the instrument}

The scientific goal of exploring the corona from 1.1 to $30 \mathrm{R}_{\mathrm{o}}$ in a single experiment requires innovation in design of the instrument, for this has never before been attempted. Furthermore, the fundamental problem of photospheric stray light rejection, common to all coronal observations, is compounded by the enormous gradient in coronal brightness with radial distance from the Sun. The total $\mathrm{K}+\mathrm{F}$ brightness varies by six orders of magnitude between 1.1 and $30 \mathrm{R}_{\mathrm{o}}$. Therefore, we have chosen to break up the observing domain into three subregions, and LASCO is composed of three compact optical systems, C1, C2 and C3, each specially designed and optimized for its particular range of operation, and all held in precise coalignment through sound mechanical and thermal design (Michels et al., 1989). Characteristics of the three optical systems are shown in Table 1. The concentric fields of view allow for extensive overlapping which is essential for intercalibration of the three systems (including cross calibration on orbit), and to assist in later reconstruction of composite wide field images. 


\subsection{OPTICAL DESIGN OF C1 $\left(1.1-3.0 \mathrm{R}_{\mathrm{o}}\right)$}

In order to image the corona with adequate spatial resolution very close to the sun's limb, an internally-occulted system is required. $\mathrm{C} 1$ implements this requirement with a reflective design, made possible by recent advances in mirror superpolishing technology. The objective mirror, M1, is an off-axis paraboloid. The solar image is formed at the prime focus, on a convex annular mirror, M2, analogous to the field lens in a standard refractive Lyot coronograph. This mirror provides a field stop, and also performs the function of the Lyot internal occulting disk, by removing the unwanted photospheric image from the system. The field mirror relays the light of the coronal image onto a second off-axis paraboloid, the collimating mirror, M3 identical to M1. This fully symmetric arrangement accomplishes complete cancellation of coma in the system. The collimated beam of coronal light is next sent through a narrow-bandpass tunable Fabry-Perot filter, and then into an objective which focuses the final coronal image onto a 1024 x 1024 pixel CCD image detector. Max-Planck Institute für Aeronomie has responsability for $\mathrm{C1}$.

The coronal light is analyzed spectroscopically by the tunable Fabry-Perot (FP) filter which is designed by the Naval Research Laboratory. The FP is an interference (comb type passband, $0.4 \AA$ FWHM) filter, which passes many interference orders. It works in conjunction with a broader blocking filter, with passband narrow enought to eliminate all but one selected order passed by the FP, but broad enough to allow tuning of the FP over a full free spectral range $(22 \AA)$. The filter presents the CCD detector with a monochromatic image of the entire $3 \mathrm{R}_{0}$ coronal field of view. Two-dimensional imaging spectroscopy of coronal line profiles is achieved by synchronizing CCD exposures with a stepwise spectral scan of the tunable filter.

\subsection{OPTICAL DESIGN OF C2 $\left(1.5-6 \mathrm{R}_{\mathrm{o}}\right)$ AND C3 $\left(3-30 \mathrm{R}_{\mathrm{o}}\right)$}

C2 and C3 are externally occulted coronographs adapted from earlier, well developped designs (SOLWIND, SMM) and optimized for their respective domain. In this design, the external occulter completely shadows the objective lens from direct sunlight, dramatically lowering the level of instrumental stray light. The main disadvantage of external occultation is that the imaging properties of the objective lens are seriously impeded by vignetting (partial obscuration of the aperture) by the shadowing assembly (i.e. the external occulter) for object points not far removed from the occulter shadow. In other words, the instrument achieves full resolution at the outer part of its field, but relatively poor resolution near the central occulter shadow. It is considerations such as this that drive the requirement for extensive overlap of all three of the optical systems.

Laboratoire d'Astronomie Spatiale has responsability for $\mathrm{C} 2$ which incorporates a new multi-thread occulter and a doublet in optical contact as the objective lens.

For C3 designed by the Naval Research Laboratory, the external occulter is a triple disk assembly, with each disk sized to intercept light diffracted from the previous one. Its objective lens is a singlet, to obtain absolutely minimal scattering. For both $\mathrm{C} 2$ and $\mathrm{C} 3$, the corona is imaged onto $1024 \times 1024$ CCD cameras. 


\section{System design}

LASCO consists of two boxes. The coronograph optical box (COB), realized by the University of Birmingham, houses the three optical systems and cameras. It is mounted to the spacecraft instrument pylon with isostatic mounting legs and an offset pointing mechanism, capable of correcting for unacceptably large errors in the spacecraft sunpointing system. The optical box is broken into two parts, one half contains the $\mathrm{C} 1$ coronograph optical train, and the other half contains the $\mathrm{C} 2$ and $\mathrm{C} 3$. A second portion of the experiment is the LASCO electronics box (LEB) which is realized by the Naval Research Laboratory. It contains microprocessors for instrument control and image processing, status and image memory, power conditioning circuitry, command and telemetry interface, etc. This box is relatively small and hard-mounted to the spacecraft.

The three telescopes are equipped with identical CCD cameras chosen because of their large dynamic range and geometric and photometric stability. Passive radiant coolers, integral to each camera package, will maintain the CCDs at the required temperatures. The three cameras are under the responsability of the Naval Research Laboratory.

TABLE 1. Characteristics of the three coronographs

\begin{tabular}{llll}
$\begin{array}{l}\text { Field of view } \\
\text { (Annular ring) }\end{array}$ & \multicolumn{1}{c}{$\mathrm{C} 1$} & \multicolumn{1}{c}{$\mathrm{C} 2$} & $\mathrm{C} 3$ \\
Optical system & $1.1-3.0 \mathrm{R}_{\mathrm{o}}$ & $1.5-6.0 \mathrm{R}_{\mathrm{o}}$ & $3-30 \mathrm{R}_{\mathrm{o}}$ \\
& $\begin{array}{l}\text { Internally } \\
\text { Occulted }\end{array}$ & $\begin{array}{l}\text { Externally } \\
\text { Occulted }\end{array}$ & $\begin{array}{l}\text { Externally } \\
\text { Occulted }\end{array}$ \\
Optical Type & Mirror & Lens & Lens \\
Pixel(angular) & $5.6 \mathrm{arc} / \mathrm{sec}$ & $11.2 \mathrm{arc} / \mathrm{sec}$ & $54 \mathrm{arc} / \mathrm{sec}$
\end{tabular}

Polarization analysis: 3 linear polarizers on each coronograph

$\begin{array}{llll}\text { Color Filters } & \text { FeXIV 530nm } & 3 \text { Broadband: } & 3 \text { Broadband: } \\ & \text { FeX 637nm } & \text { Orange } & \text { Orange } \\ & \text { CaXV 569nm } & \text { Red } & \text { Red } \\ & \text { Fraunhofer } & \text { Blue } & \text { Blue } \\ & \text { Orange } & \text { H-alpha } & \text { H-alpha }\end{array}$

\footnotetext{
References

Michels, D.J. et al. (1989): 'LASCO - A wide-field white light and spectrometric coronograph for SOHO', ESA SP-1104, 55-62.
} 